\title{
Changes in the levels of IL-1ß, cortisol and chromogranin A in saliva of subjects with occupational fatigue
}

\author{
MIN DING $^{1 *}$, CHAOXIAN ZHAO $^{2 *}$, YAN LI $^{3}$, XIAOXIA LIU ${ }^{2}$, XUELING WANG ${ }^{1}$, FENGLI LIU $^{4}$, \\ JIAN WANG ${ }^{2}$, NANYAN XIONG ${ }^{2}$, YONGHONG SONG $^{2}$ and YANLI XU ${ }^{4}$ \\ ${ }^{1}$ Central Laboratory, Medical College, Hebei University of Engineering, Handan, Hebei 056038; \\ ${ }^{2}$ Department of Biochemistry, Medical College, Hebei University of Engineering; \\ ${ }^{3}$ Emergency Department, Affiliated Hospital of Hebei University of Engineering, Handan, Hebei 056002; \\ ${ }^{4}$ Department of Epidemiology, Medical College, Hebei University of Engineering, Handan, Hebei 056038, P.R. China
}

Received April 23, 2019; Accepted April 6, 2020

DOI: $10.3892 /$ etm.2020.8799

\begin{abstract}
Changes in the levels of interleukin-1 $\beta$ (IL-1 $\beta$ ), cortisol and chromogranin A $(\mathrm{CgA})$ in saliva of subjects with occupational fatigue were investigated. Doctors in the Emergency Department were selected as research subjects. Saliva was collected before work (after full rest) and after work ( $\geq 24 \mathrm{~h}$ ). Electroencephalogram (EEG) was performed. Enzyme-linked immunosorbent assay (ELISA) was used to detect the levels of IL-1 $\beta$, cortisol and CgA in saliva. In order to obtain permission for human specimens, the study was approved by the Ethics Committee of the Affiliated Hospital of Hebei University of Engineering and registered for clinical trials (registration no. ChiC-TR-DCD-14005746). As there were only 4 subjects in this study without fatigue waves in EEG, and the number of these subjects was not sufficient to constitute a control group, the comparison of the contents of IL-1 $\beta$, cortisol and $\mathrm{CgA}$ of all subjects before and after working for $18 \mathrm{~h}$ was just a confirmation of the statistical results of 43 cases with fatigue waves in the EEG. According to the results, there was no change in the contents of IL- $1 \beta$ and cortisol in the saliva of subjects with occupational fatigue before and after fatigue, whereas, there was a significant change in the content of $\mathrm{CgA}$ before and after fatigue. However, there was no correlation between the content of $\mathrm{CgA}$ and fatigue. The results of the present study revealed that $\mathrm{IL}-1 \beta$, cortisol and $\mathrm{CgA}$ indicators are not suitable diagnostic markers for occupational fatigue.
\end{abstract}

Correspondence to: Dr Yanli Xu, Department of Epidemiology, Medical College, Hebei University of Engineering, 19 Taiji Road, Handan Economic and Technological Development Zone, Handan, Hebei 056038, P.R. China

E-mail: xu_yanli9@163.com; xuyanli@vip.sina.com

*Contributed equally

Key words: occupational fatigue, saliva, interleukin-1 $\beta$, cortisol, chromogranin A

\section{Introduction}

Fatigue is a complex state of psychophysiology. Although the study of fatigue has been carried out since the early 20th century, there is still no accurate definition and there are differences in the classification of fatigue. Fatigue is generally divided into physical and mental fatigue.

Fatigue may lead to loss of attention and mood disorders, and reduce work efficiency (1). Some of the higher-risk, stressful occupations, such as pilots, drivers, police and medical staff, are prone to these symptoms. Mental and physical fatigue is one of the major public health problems worldwide. The annual global injury caused by fatigue accounts for $21.7 \%$ of occupational injuries (2) and the deaths caused by fatigue driving account for 57\% of traffic deaths; especially the accidents of coach on highway caused by fatigue are $>7$ times the ordinary accidents (3). In China, 600,000 individuals die each year due to fatigue accidents (4).

In China, the clinical doctors in the Emergency Department not only work continuously for a long time, but also work with great intensity; they often do not get enough rest to relieve fatigue and are prone to occupational fatigue. Occupational fatigue not only damages their health, but also threatens the medical level and quality of care for patients. Since the World Health Organization listed fatigue as one of the main factors that threaten human health in the 21st century, fatigue has gradually attracted widespread attention in the research field (5-9). Therefore, the identification of markers of fatigue and the timely monitoring of vulnerable individuals can greatly prevent injuries to themselves and prevent harm to other individuals due to occupational fatigue.

The methods of assessing fatigue can be divided into subjective and objective assessments. Due to the non-specificity and subjectivity of fatigue, the earliest research on fatigue is almost subjective (mostly fatigue scale); however, subjective assessment cannot avoid the inherent low inefficiency and reliability. For a deeper understanding and more accurate measurement of fatigue, intervention of physiological and biochemical indicators is essential.

Physiological indicators of fatigue are mainly electrophysiological indicators, such as electroencephalogram (EEG), 
electrooculogram, electrocardiogram and event-related potentials, with EEG being the most studied one (10). It is generally believed that the $\alpha$ wave is the basic rhythm of normal human brain wave. When the cerebral cortex is excited, $\beta$ waves appear. Appearance of $\theta$ waves is generally a manifestation of nervous system inhibition. As early as 1993, Brookhuis and de Waard (11) used the brain energy parameter $(\theta+\alpha) / \beta$ to assess driver status and found that the parameter showed an upward trend with the increase of working time and fatigue state. Hypothalamic-pituitary-adrenal (HPA) is an important part of the neuroendocrine system, which mainly maintains homeostasis and response to stress. Activation of HPA is an adaptive protective response to external stress. When the body is under stress, hypothalamus promotes corticotrophin to release hormone secretion, activates adrenal cortex function and elevates the hormone level of cortisol in serum. Therefore, some scholars proposed that EEG is the most reliable indicator of clinical psychological fatigue (12). However, in practical terms, the measurement of EEG requires expensive equipment and the procedure is quite complicated. Therefore, the research on EEG is almost entirely limited to laboratory research. At the same time, the collection of biochemical indicators involves invasive techniques. Therefore, as a tool to quickly evaluate fatigue, the biochemical indicators are not suitable in a number of occasions. With the advancement of society and the continuous improvement of cultural living standards, there is an increasing demand for medical examinations and diagnostic methods that are non-invasive, simple and rapid. Therefore, in recent years, saliva specimens have received widespread attention from researchers. Compared with serum samples, saliva collection is safe and convenient, non-invasive, and free of the risk of transmission of blood-borne diseases. For patients, saliva collection is painless and easy to accept. Saliva has the advantage of being able to be sampled in real time compared with urine specimens. Saliva detection research has attracted great interest and achieved some initial results. Our preliminary experiments (13) showed that salivary components under finite fatigue conditions can obtain good peptide spectrum recognition in the range of 2,000-15,000 Da during time-of-flight mass spectrometry detection, and it was shown that the detection of multiple peptides and fatigue presented certain correlations. Through a series of statistical analyses, the following fatigue-related proteins were identified: Ig $\kappa$ chain V-I region Lay, growth factor receptor-bound protein 2, ADP-ribosyl cyclase/cyclic ADP-ribose hydrolase 2, lipocalin-1, mitochondrial malate dehydrogenase, heat shock cognate $71 \mathrm{kDa}$ protein, Ig $\kappa$ chain V-III region SIE, Golgi membrane protein 1, cystatin-A, mucin-19, heat shock protein $\beta-1$, Ig $\gamma-3$ chain $C$ region, Serpin B13, Rab GDP dissociation inhibitor $\beta$, Annexin A1, afamin, protein disulfide-isomerase $\mathrm{A} 3, \mathrm{Ig} \kappa$ chain $\mathrm{V}$-III region $\mathrm{HAH}$, transmembrane protease serine $11 \mathrm{D}$, nucleoside diphosphate kinase $\mathrm{B}$, bactericidal permeability-increasing protein, ubiquitin-like modifier-activating enzyme 1, macrophage-capping protein, myeloid-derived growth factor, pancreatic $\alpha$-amylase, L-lactate dehydrogenase B chain, mitochondrial peroxiredoxin-5, prominin-1, type II cytoskeletal keratin 4, and salivary acidic proline-rich phosphoprotein 1/2 (13).

Occupational fatigue is not a simple physical or mental fatigue. The mechanism of occupational fatigue is very complicated. It is all-embracing and there is no unified fatigue theory. At the same time, due to the multiple factors of fatigue and their mutual influence, the fatigue factor theory of single factor is also unscientific. Numerous hypotheses about the mechanism of fatigue have been proposed, such as the HPA axis imbalance (14). Exercise fatigue stress activates the HPA axis and the sympathetic nervous system, leading to the activation of immune cells (mainly microglia and astrocytes) in the brain and the secretion of a large number of inflammatory factors [such as interleukin-1 $\beta$ (IL-1 $\beta$ ) and tumor necrosis factor $\alpha$, resulting in neuroinflammation. IL-1 $\beta$ is a molecular of IL-1 composed of 153 amino acids, and almost all nucleated cells secrete IL-1 $\beta$. IL-1 $\beta$ is one of the most potent inflammatory mediators in the body and has a number of biological functions. Under normal circumstances, IL-1 $\beta$ is at low levels in the central nervous system and plays an immunomodulatory role, such as promoting T-cell differentiation and B-cell proliferation. When the body is under stress, the hypothalamus promotes the secretion of corticotropin-releasing hormones, activates the adrenal function and raises the serum cortisol levels. Cortisol is a neuroendocrine hormone involved in the metabolism of sugar, fat and protein. It has anti-inflammatory, anti-allergic, immunosuppressive effects, activates the nervous system vitality, and regulates cardiovascular system function. Cortisol affects sleep patterns, mood, cognition and sensation through the blood-brain barrier. In the fatigue state, small molecules, such as salivary gangliosides and cortisol, can be changed regularly; however, it is difficult to establish a convenient detection system.

Chromogranin A (CgA) is an acidic hydrophilic secreted protein in the chromaffin-like particles of neuroendocrine cells. $\mathrm{CgA}$ was originally discovered in the secretory granules of chromaffin cells of the adrenal gland. In the adrenal medullary chromaffin particles, which are co-secreted with catecholamines and calcium, $\mathrm{CgA}$ can also reflect the function of the adrenal cortex to some extent. Therefore, in the present study, it was speculated that changes in the levels of IL-1 $\beta$, cortisol and $\mathrm{CgA}$ in saliva are associated with fatigue.

Doctors in the Emergency Department were selected as research subjects as they have a long working time and labor intensity, and are more prone to fatigue. Saliva detection is a detection method that has emerged in recent years. It is characterized by non-invasiveness, convenience, and less cost, and has attracted the attention of scholars in disease diagnosis and prognosis, drug detection, and physical and psychological stress evaluation. In the present study, IL-1 $\beta$, cortisol and $\mathrm{CgA}$ in saliva were used as indicators to detect whether there were any changes in the three indexes after work and recovery. The analysis of fatigue markers in saliva provides reference for the establishment and verification of the fatigue-saliva marker detection model and the rapid detection of fatigue with saliva.

\section{Subjects and methods}

General information. From December 2015 to March 2016, a total of 56 emergency physicians were selected, according to specific inclusion and exclusion criteria, from the Affiliated Hospital of Hebei University of Engineering (Handan, China), including 28 males and 28 females, 30-49 years of age. The CRP and cholesterol levels of subjects were within the normal 
range. In total, 47 pairs of samples were successfully collected, including samples from 24 males and 23 females. Among the remaining 9 subjects, samples were not successfully collected from 4 cases, due to insufficient saliva secretion, and 5 were lost after work due to departmental rotation. Of the 47 subjects, there were 4 subjects ( 3 males and 1 female) without fatigue waves in the EEG after working for $18 \mathrm{~h}$. Saliva was collected before work (after full rest) and after work ( $\geq 24 \mathrm{~h}$ ). The study was approved by the Ethics Committee of the Affiliated Hospital of Hebei University of Engineering and registered (registration no. ChiC-TR-DCD-14005746). All subjects who participated in this research signed an informed consent and had complete clinical data.

Inclusion and exclusion criteria of research subjects. Inclusion criteria: Healthy subjects who volunteered to participate in the study and signed an informed consent; with no oral diseases, no organic diseases or chronic fatigue symptoms; who received no medications or dietary supplements in the past 3 months. Exclusion criteria: i) Subjects with sustained or recurrent fatigue that lasted $>6$ months, with sore throat, swollen neck or axillary lymph nodes, muscle pain, or multiple non-arthritic pain; ii) with headache; iii) with sleep disorder; iv) with discomfort that persisted $>24 \mathrm{~h}$ after exertion; v) with hypoglycemia, heart disease, or vi) subjects recently under diet to lose weight.

Collection and preservation of saliva. Before sampling, the participants were asked to use distilled water to wash their mouth in order to remove any food residues (15). All subjects were asked to sit vertically in front of the mirror for $5 \mathrm{~min}$ after squatting. Eyes and mouth were kept open, the tongue kept touching with palate, and saliva was let to flow down the lower lip. Fresh saliva was stored in a 2-ml saliva collection tube. If the amount of saliva was insufficient, saliva collection tube was used to gently stimulate the lower lip to increase the secretion of saliva. Doctors' saliva samples were collected in their spare time after shift, and were marked every morning. Samples before work (before night shift) were marked as 'A', samples after work (after night shift) were marked as 'B', and samples after recovery (before next day work) were marked as ' $\mathrm{C}$ ', and they were marked in the form of 'personnel number-fatigue status number'. All samples were sent to the Laboratory of the Medical School of Hebei University of Engineering and stored in a $-70^{\circ} \mathrm{C}$ refrigerator. The Emergency Department was close to the Laboratory and the samples were collected in winter with a temperature below $0^{\circ} \mathrm{C}$, thus no insulation was used outside.

\section{Indicator detection}

EEG data collection. After a long period of work $(\geq 18 \mathrm{~h})$ and after collecting saliva, doctors were subjected to EEG to detect the presence of fatigue waves (16) (slow $\delta$ - and $\theta$-wave increase, and fast $\alpha$ - and $\beta$-wave decrease). SOLAR-RTA and SOLAR-BFM brain function monitoring system (SOLAR Electronic Technology Co., Ltd.) was used in video EEG monitoring and EEG acquisition system. Real-time analysis of EEG function, comprehensive analysis of brain function, and analysis of leading volatility trend of brain function vital signs were carried out (ISO9001 and ISO13485 international quality management system certification). EEG was recorded for at least $30 \mathrm{~min}$. Silver disc electrodes were placed in conventional manner. Scalp record point was 16 . The silver plate electrode was used as the recording electrode. The sampling rate was filtered, each record was not $<30 \mathrm{~min}$, and each case was recorded at least once.

Biochemical indicator detection. Quantikine ${ }^{\circledR} \mathrm{HS}$ Enzyme-linked immunosorbent assay (ELISA) Human IL-1 $\beta /$ IL-1F2 Immunoassay (cat. no. HSLB00D) and Parameter $^{\mathrm{TM}}$ Cortisol Assay (cat. no. KGE008B) (both from R\&D Systems, Inc.) were used. Human CgA ELISA kit was purchased from Shanghai Jianglai Biotechnology Co., Ltd. The detection of $\mathrm{pH}$ values was performed using RAPIDPoint 405 Blood Gas Analyzer produced by Siemens AG.

Quality control. Members of the research team were trained before the clinical trial. Volunteers were informed in detail about the clinical trial and signed an informed consent form. Volunteers were numbered.

Detection methods. Saliva samples were taken from the $-70^{\circ} \mathrm{C}$ refrigerator, thawed at room temperature, and centrifuged at $10,000 \mathrm{xg}$ at $4^{\circ} \mathrm{C}$ for $5 \mathrm{~min}$. The levels of $\mathrm{CgA}, \mathrm{IL}-1 \beta$, cortisol and $\mathrm{pH}$ in pre-fatigue and post-fatigue samples were determined according to the manufacturer's instructions.

Statistical analysis. Continuous variables were tested for normal distribution. For the non-normally distributed data, Wilcoxon test was used to detect whether the index difference between before and after working for $18 \mathrm{~h}$ was significant in all subjects and the subjects in the fatigue wave group. Spearman's correlation analysis was performed on the differences in the levels of IL-1 $\beta$, cortisol and $\mathrm{CgA}$ before and after work to explore the correlation with the appearance of fatigue waves in the EEG. ROC curve analysis was used to analyze the predictive value of these three indicators for fatigue. All analyses were performed using SPSS 17.0 software (IBM Corp.). $\mathrm{P}<0.05$ was considered to indicate a statistically significant difference.

\section{Results}

Levels of $I L-1 \beta$, cortisol and $C g A$ in fatigue wave group and all research subjects. There was no significant difference in the content of IL-1 $\beta$ or cortisol in the subjects of the fatigue wave group, between before and after working for $18 \mathrm{~h}(\mathrm{P}>0.05)$. However, there was a statistically significant difference in the content of $\mathrm{CgA}$ in the subjects of the fatigue wave group between before and after working for $18 \mathrm{~h}(\mathrm{P}<0.05$; Table I).

There was no significant difference in the content of IL-1 $\beta$ or cortisol in all subjects between before and after working for $18 \mathrm{~h}(\mathrm{P}>0.05)$. However, there was a significant difference in the content of $\mathrm{CgA}$ in all subjects between before and after working for $18 \mathrm{~h}(\mathrm{P}<0.05$; Table II).

Correlation of $I L-1 \beta$, cortisol and $C g A$ with fatigue waves in $E E G$. Spearman's correlation analysis was performed to examine the correlation between the IL- $1 \beta$ difference of all subjects before and after work and whether there were 
Table I. Comparison of three indicators before and after $18 \mathrm{~h}$ of work in the fatigue wave group (n=43) $[M(Q R)]$.

\begin{tabular}{lccc}
\hline Time & IL-1 $\beta$ & Cortisol & CgA \\
\hline Before work & $25.816(14.108,75.763)$ & $5.815(2.465,13.785)$ & $274.596(238.373,289.7016)$ \\
After work & $30.942(5.966,66.528)$ & $7.64(2.28,17.49)$ & $254.523(220.592,286.965)$ \\
Z value & 0.181 & 0.193 & 2.174 \\
P-value & 0.856 & 0.847 & 0.030 \\
\hline
\end{tabular}

IL-1 $\beta$, interleukin-1 $\beta ; \mathrm{CgA}$, chromogranin A.

Table II. Comparison of three indicators before and after $18 \mathrm{~h}$ of work in all research subjects $(\mathrm{n}=47)[\mathrm{M}(\mathrm{QR})]$.

\begin{tabular}{lccc}
\hline Time & IL-1 $\beta$ & Cortisol & CgA \\
\hline Before work & $24.9229(14.1083,72.5811)$ & $5.8150(2.4650,13.7850)$ & $269.17(238.37,289.70)$ \\
After work & $30.5769(5.9282,59.7377)$ & $7.6400(2.2800,17.6650)$ & $254.86(220.59,286.96)$ \\
Z value & 0.455 & 0.677 & 2.116 \\
P-value & 0.649 & 0.498 & 0.034 \\
\hline
\end{tabular}

IL-1 $\beta$, interleukin-1 $\beta ; \mathrm{CgA}$, chromogranin A.

fatigue waves in EEG. The results showed that the correlation coefficient was $\mathrm{r}=-0.158, \mathrm{P}=0.288$. In addition, Spearman's correlation analysis on the cortisol difference of all subjects before and after work and whether there were fatigue waves in EEG showed that the correlation coefficient was $r=0.139$, $\mathrm{P}=0.351$; and for the $\mathrm{CgA}$ difference of all subjects before and after work and whether there was fatigue waves in EEG, the results showed that the correlation coefficient was $r=-0.135$, $\mathrm{P}=0.364$.

Diagnostic value of the difference of $I L-1 \beta$, cortisol and $C g A$ content before and after work. The existence of fatigue waves in EEG was used as the gold standard to analyze the diagnostic value of the difference of IL-1 $\beta$ content before and after work. The results for IL-1 $\beta$ showed that the area under the ROC curve was 0.634 (Fig. 1), for cortisol the area under the ROC curve was 0.262 (Fig. 2), and for $\mathrm{CgA}$ the area under the ROC curve was 0.366 (Fig. 3).

\section{Discussion}

Recent neuropathological studies have shown that glial cell-mediated neuroinflammation leads to a decline in learning and memory (17-19). IL-1 $\beta$ is a molecular form of IL-1 composed of 153 amino acids, and almost all nucleated cells secrete IL-1 $\beta$. IL-1 $\beta$ is one of the most potent inflammatory mediators in the body and has a wide range of biological functions. Under normal circumstances, IL-1 $\beta$ is at low levels in the central nervous system and plays an immunomodulatory role, such as promoting T-cell differentiation and B-cell proliferation. When the body receives a stress response, IL-1 $\beta$ levels rise and produce toxic effects. In 1993, a study on patients with prostate cancer (20) showed that the severe fatigue was always accompanied by high plasma IL-1 $\beta$ levels

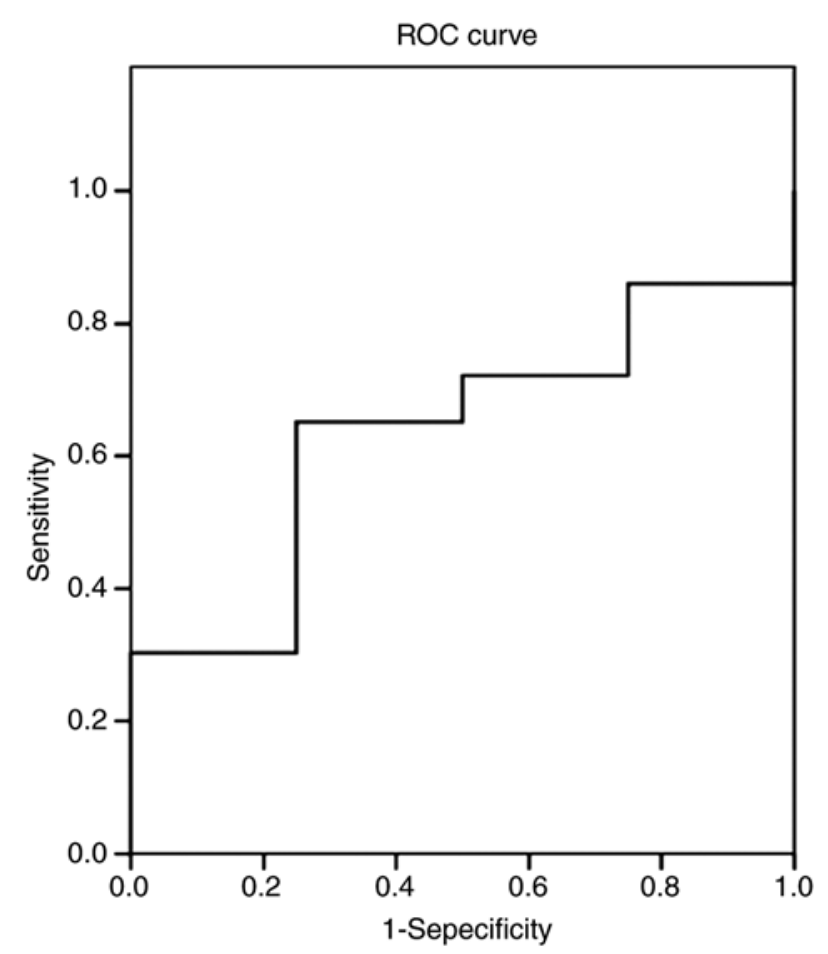

Figure 1 . ROC curve for interleukin- $1 \beta$ content. The area under the ROC curve was 0.634 .

during radiation therapy. Another study revealed that excessive immune activation has a detrimental effect on learning and memory, and IL-1 $\beta$ is a key mediator in this process (21). Baum et al (22) have also shown that after 12 weeks of training, the synthesis of IL- $1 \beta$ and IL- 6 in the plasma of cross-country runners increased. These studies have shown that exercise fatigue leads to an increase in inflammatory 


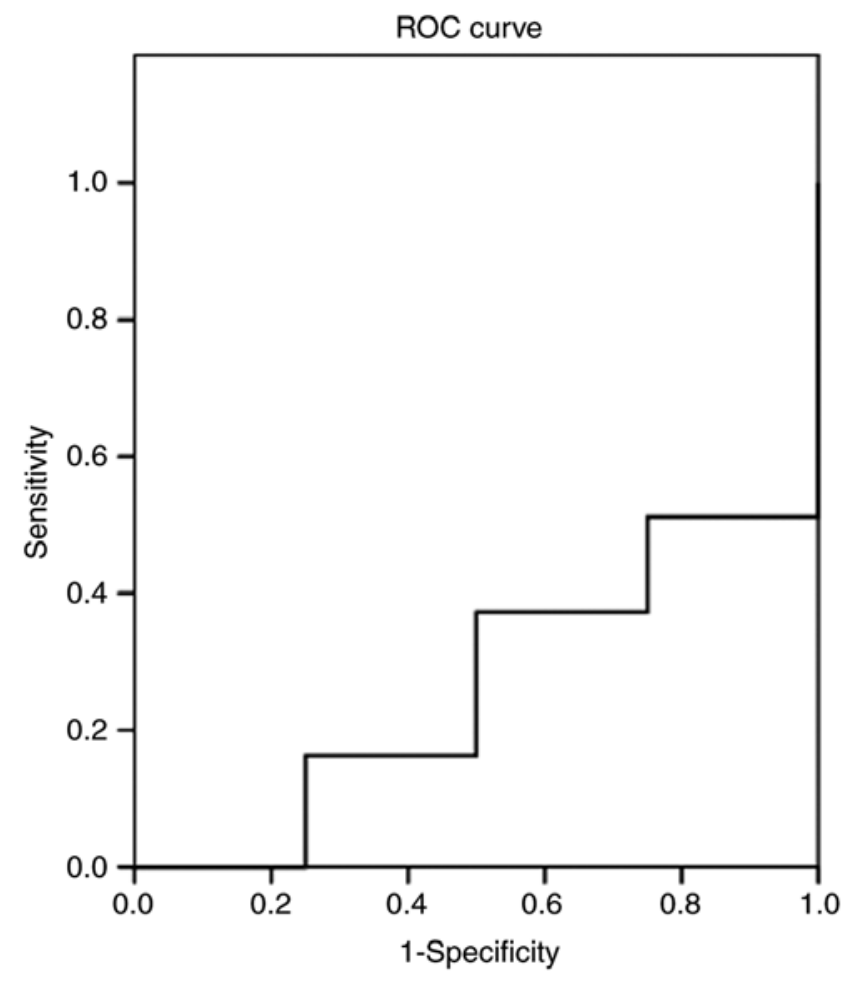

Figure 2. ROC curve for cortisol. The area under the ROC curve was 0.262.

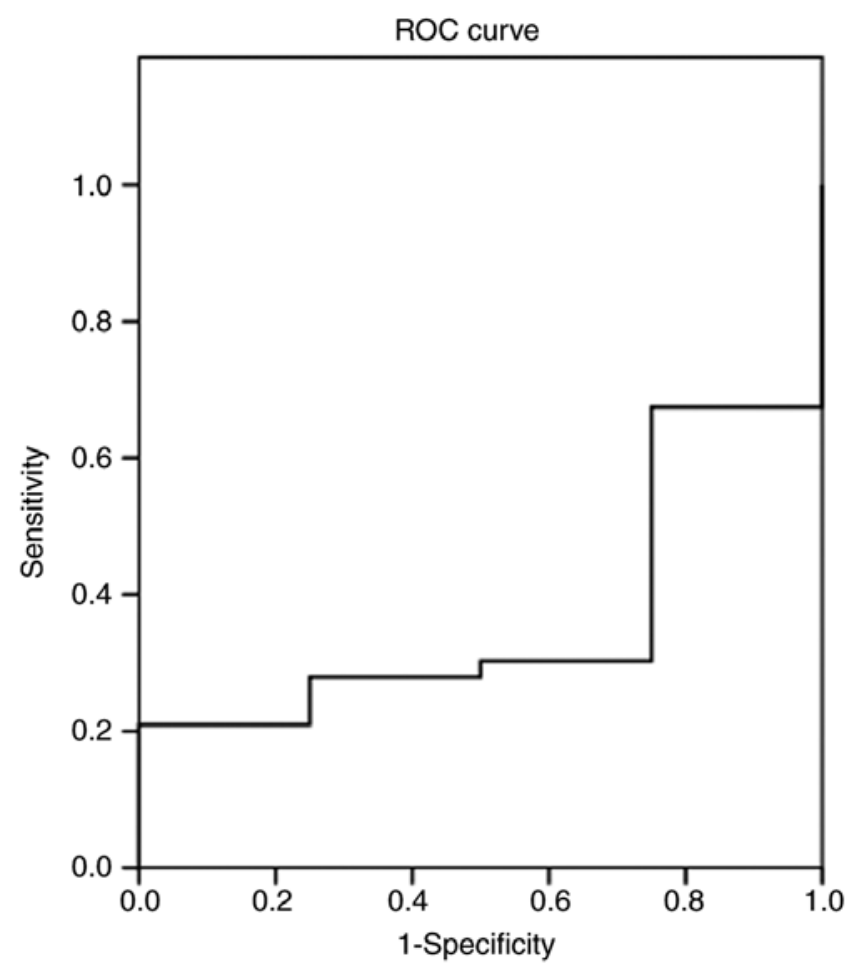

Figure 3. ROC curve for chromogranin A. The area under the ROC curve was 0.366 .

factors in peripheral tissues. In addition, it has been reported that exercise fatigue can directly lead to elevated levels of inflammatory factors in the brain (23). Further research has revealed that exercise-induced inflammatory responses are first mediated by the HPA axis, and inflammatory factors can also stimulate the HPA axis, causing elevated levels of glucocorticoids, such as adrenal ketones, to affect inflammation and immune responses $(24,25)$. In the present study, there was no significant difference in the IL- $1 \beta$ content of subjects in the fatigue wave group before and after working for $18 \mathrm{~h}(\mathrm{P}>0.05)$, which is different from previous studies $(20,22)$. This may be because the present research focused on emergency doctors and comprehensive fatigue, but not simple exercise fatigue or cancer-induced fatigue. Moreover, previous studies have mainly detected the level of IL-1 $\beta$ in plasma, whereas in the present study the changes in the content of IL-1 $\beta$ in saliva were detected. There was no significant difference in the content of IL-1 $\beta$ in all 47 subjects before and after working for $18 \mathrm{~h}$ $(\mathrm{P}>0.05)$. As there were only 4 subjects in the study without fatigue waves in EEG, and the number of these subjects was not sufficient to constitute a control group, the comparison of the contents of IL-1 $\beta$, cortisol and $\mathrm{CgA}$ of all subjects before and after working for $18 \mathrm{~h}$ was just a confirmation of the statistical results of 43 cases with fatigue waves in the EEG.

Spearman's correlation analysis was performed on the IL-1 $\beta$ difference of all subjects before and after work and whether there was fatigue waves in EEG. The results showed that the correlation coefficient was $\mathrm{r}=-0.158, \mathrm{P}=0.288$. The existence of fatigue waves in EEG was used as the gold standard to analyze the diagnostic value of the difference of IL-1 $\beta$ content before and after work. The results showed that the area under the ROC curve was 0.634 . This may be due to the fact that in the presented study whole saliva was collected, which was susceptible to the external environment. In addition, the saliva samples in this study were concentrated by high-speed centrifugation to effectively detect the components, which may have affected the sensitivity and specificity of the detection. Therefore, the saliva preparation method should be improved in order to reduce the loss of fatigue markers in saliva, to reflect objectively the content of fatigue markers in saliva, and effectively detect the low content of fatigue markers, leading to the identification of fatigue markers. In addition, saliva samples can be pre-graded according to the molecular weight of fatigue markers to improve the detection rate of fatigue markers. These are the keys to our future study.

Cortisol is a neuroendocrine hormone that participates in the body's metabolism of sugar, fat and protein, and has anti-inflammatory, anti-allergic and immunosuppressive effects. Cortisol can activate the nervous system vitality and regulate the cardiovascular system function. Cortisol affects sleep patterns, mood, cognition, and sensation through the blood-brain barrier. Salivary cortisol can reflect the level of biologically active free cortisol in the blood (26). Compared with blood tests, saliva detection has the advantages of non-invasion, convenience, and high re-sampling ability, and no sharp increase in cortisol content caused by stress. Cortisol circadian rhythm can reflect the rhythm characteristics of the HPA axis, and has become a common indicator to study circadian rhythm $(27,28)$. However, when studying the role of HPA axis in Chronic Fatigue Syndrome (CFS), the conclusions of different studies are not consistent. Moorkens et al (29) collected samples from 22 cases of CFS patients and 9 healthy controls, 5 times from 22:00 to 06:00 to detect cortisol, and a peak in CFS patients was shown to decrease. Hamilos et al (30) collected blood samples 7 times 
in $24 \mathrm{~h}$ and the same results were obtained. MacHale et al (31) collected blood samples from 30 patients with CFS and 15 healthy subjects during the day and night, and found no significant difference in cortisol levels between CFS patients and healthy subjects. Papanicolaou et al (32) found no difference in 6 sampling tests within $24 \mathrm{~h}$. Inconsistent results were also obtained from saliva tests (33-36). In the present study, there was no significant difference in the cortisol content of 43 subjects in the fatigue wave group before and after working for $18 \mathrm{~h}(\mathrm{P}>0.05)$ and neither was any significant difference in the cortisol content of all 47 subjects before and after working for $18 \mathrm{~h}(\mathrm{P}>0.05)$.

Spearman's correlation analysis was performed on the cortisol difference of all subjects before and after work and whether there was fatigue waves in EEG. The results revealed that the correlation coefficient was $\mathrm{r}=0.139, \mathrm{P}=0.351$. Whether there were fatigue waves in EEG was used as the gold standard to analyze the diagnostic value of the difference of cortisol content before and after work. The results showed that the area under the ROC curve was 0.262 . This may be because our research is self-controlled, and there is no control group for non-fatigue. Perhaps the emergency doctors need long-term night shift and circadian rhythm is destructed, and total amount of cortisol in saliva is different from non-fatigue subjects, which requires a more in-depth research. Moreover, cortisol content in saliva collected at regular intervals can only reflect cortisol level in a short period of time, and cannot reflect the overall situation of cortisol secretion during long-term occupational fatigue. Multi-time sampling will complicate the sample collection process, which will result in difficulty in sample collection, affect the subject compliance, and cause errors in sampling time. Therefore, the present study only sampled before and after fatigue. Schmidt et al (37) examined salivary cortisol levels in 265 breast cancer patients undergoing adjuvant therapy, 5 times a day, and showed that physical fatigue was not associated with morning cortisol level, cortisol response after waking, or the slope of cortisol. In addition, emotional and cognitive fatigues were not associated with any cortisol parameters. The present study, revieled as well that the difference in cortisol between before and after fatigue was not related to the presence of fatigue waves in EEG, further confirming that the cortisol content in saliva is of no value in the diagnosis of occupational fatigue.

There is no report on the relationship between $\mathrm{CgA}$ and fatigue. There was a statistically significant difference in the content of $\mathrm{CgA}$ in the 43 subjects in the fatigue wave group before and after working for $18 \mathrm{~h}(\mathrm{P}<0.05)$. There was a statistically significant difference in $\mathrm{CgA}$ content in all 47 subjects before and after working for $18 \mathrm{~h}(\mathrm{P}<0.05)$, which further proved our hypothesis.

Spearman's correlation analysis was also performed on the $\mathrm{CgA}$ difference of all subjects before and after work and whether there were fatigue waves in EEG. The results showed that the correlation coefficient was $\mathrm{r}=-0.135, \mathrm{P}=0.364$. Whether there was fatigue waves in EEG was used as the gold standard to analyze the diagnostic value of the difference of $\mathrm{CgA}$ content before and after work. The results showed that the area under the ROC curve was 0.366. At the same time, as a protein molecule, chromophorin is superior to cortisol in immunogenicity. Our study is a preliminary study for the rapid detection of $\mathrm{CgA}$ as a biomarker of fatigue by colloidal gold and other methods.

In this study, there was no control group and the comparison of fatigue before and after work of subjects was investigated. In addition, the relationship between blood and salivary levels of IL-1 $\beta$, cortisol and CgA were not analyzed. At the same time, the number of samples tested was relatively small. More subjects with different backgrounds should be enrolled to develop a simple and quick detection method of fatigue.

\section{Acknowledgements}

Not applicable.

\section{Funding}

The study was supported by the National Natural Science Foundation of China (project no. 81373095).

\section{Availability of data and materials}

The datasets used and/or analyzed during the present study are available from the corresponding author on reasonable request.

\section{Authors' contributions}

MD and $\mathrm{CZ}$ wrote the manuscript. MD and YX performed ELISA. YL and XL collected and analyzed the general data of the research subjects. $\mathrm{CZ}$ and $\mathrm{XW}$ were responsible for the detection of the biochemical indicators. FL, YS and JW assisted with statistical analysis. NX collected saliva samples. All authors read and approved the final manuscript.

\section{Ethics approval and consent to participate}

The study was approved by the Ethics Committee of the Affiliated Hospital of Hebei University of Engineering (Handan, China). All subjects who participated in this research signed an informed consent and had complete clinical data.

\section{Patient consent for publication}

Not applicable.

\section{Competing interests}

The authors declare that they have no competing interests.

\section{References}

1. Lal SK and Craig A: Driver fatigue: Electroencephalography and psychological assessment. Psychophysiology 39: 313-321, 2002.

2. Arlinghaus A, Lombardi DA, Willetts JL, Folkard S and Christiani DC: A structural equation modeling approach to fatigue-related risk factors for occupational injury. Am J Epidemiol 176: 597-607, 2012.

3. Philip P, Sagaspe P, Moore N, Taillard J, Charles A, Guilleminault C and Bioulac B: Fatigue, sleep restriction and driving performance. Accid Anal Prev 37: 473-478, 2005.

4. Zhang G, Yau KK, Zhang X and Li Y: Traffic accidents involving fatigue driving and their extent of casualties. Accid Anal Prev 87: 34-42, 2016. 
5. Janssen $\mathrm{N}$ and Nijhuis FJ: Associations between positive changes in perceived work characteristics and changes in fatigue. J Occup Environ Med 46: 866-875, 2004.

6. de Croon EM, Blonk RW, de Zwart BC, Frings-Dresen MH and Broersen JP: Job stress, fatigue, and job dissatisfaction in Dutch lorry drivers: Towards an occupation specific model of job demands and control. Occup Environ Med 59: 356-361, 2002.

7. Bultmann U, Kant IJ, Schroer CA and Kasl SV: The relationship between psychosocial work characteristics and fatigue and psychological distress. Int Arch Occup Environ Health 75: 259-266, 2002.

8. van der Ploeg E and Kleber RJ: Acute and chronic job stressors among ambulance personnel: Predictors of health symptoms. Occup Environ Med 60 (Suppl 1): i40-i46, 2003.

9. Dimsdale JE, Ancoli-Israel S, Elsmore TF and Gruen W: Taking fatigue seriously: I. Variations in fatigue sampled repeatedly in healthy controls. J Med Eng Technol 27: 218-222, 2003.

10. Cockram MS, Murphy E, Ringrose S, Wemelsfelder F, Miedema HM and Sandercock DA: Behavioural and physiological measures following treadmill exercise as potential indicators to evaluate fatigue in sheep. Animal 6: 1491-1502, 2012.

11. Brookhuis KA and de Waard D: The use of psychophysiology to assess driver status. Ergonomics 36: 1099-1110, 1993.

12. Shen K, Li X, PM Pullens W, Zheng H, Ong CJ and V Wilder-Smith E: Key feature extraction for fatigue identification using random forests. Conf Proc IEEE Eng Med Biol Soc 2: 2044-2047, 2005.

13. Xu YL, Gong YN, Xiao D, Zhao CX, Gao XH, Peng XH, Xi AP, He LH, Lu LP, Ding M, et al: Discovery and identification of fatigue-related biomarkers in human saliva. Eur Rev Med Pharmacol Sci 22: 8519-8536, 2018.

14. Bower JE, Ganz PA, Dickerson SS, Petersen L, Aziz N and Fahey JL: Diurnal cortisol rhythm and fatigue in breast cancer survivors. Psychoneuroendocrinology 30: 92-100, 2005.

15. Nunes LA, Brenzikofer R and Macedo DV: Reference intervals for saliva analytes collected by a standardized method in a physically active population. Clin Biochem 44: 1440-1444, 2011.

16. Lal SK, Craig A, Boord P, Kirkup L and Nguyen H: Development of an algorithm for an EEG-based driver fatigue countermeasure. J Safety Res 34: 321-328, 2003.

17. Srinivasan D, Yen JH, Joseph DJ and Friedman W: Cell type-specific interleukin-1beta signaling in the CNS. J Neurosci 24: 6482-6488, 2004.

18. Wang DS, Zurek AA, Lecker I, Yu J, Abramian AM, Avramescu S, Davies PA, Moss SJ, Lu WY and Orser BA: Memory deficits induced by inflammation are regulated by $\alpha 5$-subunit-containing GABAA receptors. Cell Rep 2: 488-496, 2012.

19. Song C, Phillips AG, Leonard BE and Horrobin DF Ethyl-eicosapentaenoic acid ingestion prevents corticosterone-mediated memory impairment induced by central administration of interleukin-1beta in rats. Mol Psychiatry 9: 630-638, 2004

20. Greenberg DB, Gray JL, Mannix CM, Eisenthal S and Carey M: Treatment-related fatigue and serum interleukin-1 levels in patients during external beam irradiation for prostate cancer. J Pain Symptom Manage 8: 196-200, 1993.

21. Rachal Pugh C, Fleshner M, Watkins LR, Maier SF and Rudy JW: The immune system and memory consolidation: A role for the cytokine IL-1beta. Neurosci Biobehav Rev 25: 29-41, 2001.
22. Baum M, Klöpping-Menke K, Müller-Steinhardt M, Liesen $H$ and Kirchner H: Increased concentrations of interleukin 1-beta in whole blood cultures supernatants after 12 weeks of moderate endurance exercise. Eur J Appl Physiol Occup Physiol 79: 500-503, 1999

23. Pervaiz $\mathrm{N}$ and Hoffman-Goetz L: Immune cell inflammatory cytokine responses differ between central and systemic compartments in response to acute exercise in mice. Exerc Immunol Rev 18: 142-157, 2012.

24. Barriga C, Martin MI, Ortega E and Rodriguez AB: Physiological concentrations of melatonin and corticosterone in stress and their relationship with phagocytic activity. J Neuroendocrinol 14: 691-695, 2002.

25. Besedovsky HO and Rey AD: Physiology of psychoneuroimmunology: A personal view. Brain Behav Immun 21: 34-44, 2007.

26. Castro M, Elias PC, Martinelli CE Jr, Antonini SR, Santiago L and Moreira AC: Salivary cortisol as a tool for physiological studies and diagnostic strategies. Braz J Med Biol Res 33 $1171-1175,2000$

27. Jerjes WK, Peters TJ, Taylor NF, Wood PJ, Wessely S and Cleare AJ: Diurnal excretion of urinary cortisol, cortisone, and cortisol metabolites in chronic fatigue syndrome. J Psychosom Res 60: 145-153, 2006.

28. Jerjes WK, Cleare AJ, Wessely S, Wood PJ and Taylor NF: Diurnal patterns of salivary cortisol and cortisone output in chronic fatigue syndrome. J Affect Disord 87: 299-304, 2005.

29. Moorkens G, Berwaerts J, Wynants H and Abs R: Characterization of pituitary function with emphasis on GH secretion in the chronic fatigue syndrome. Clin Endocrinol (Oxf) 53: 99-106, 2000.

30. Hamilos DL, Nutter D, Gershtenson J, Redmond DP, Clementi JD, Schmaling KB, Make BJ and Jones JF: Core body temperature is normal in chronic fatigue syndrome. Biol Psychiatry 43: 293-302, 1998

31. MacHale SM, Cavanagh JT, Bennie J, Carroll S, Goodwin GM and Lawrie SM: Diurnal variation of adrenocortical activity in chronic fatigue syndrome. Neuropsychobiology 38: 213-217, 1998.

32. Papanicolaou DA, Amsterdam JD, Levine S, McCann SM, Moore RC, Newbrand CH, Allen G, Nisenbaum R, Pfaff DW, Tsokos GC, et al: Neuroendocrine aspects of chronic fatigue syndrome. Neuroimmunomodulation 11: 65-74, 2004.

33. Wood B, Wessely S, Papadopoulos A, Poon L and Checkley S: Salivary cortisol profiles in chronic fatigue syndrome. Neuropsychobiology 37: 1-4, 1998.

34. Strickland P, Morriss R, Wearden A and Deakin B: A comparison of salivary cortisol in chronic fatigue syndrome, community depression and healthy controls. J Affect Disord 47: 191-194, 1998.

35. Gaab J, Huster D, Peisen R, Engert V, Schad T, Schurmeyer TH and Ehlert U: Low-dose dexamethasone suppression test in chronic fatigue syndrome and health. Psychosom Med 64: 311-318, 2002 .

36. Cleare AJ: The neuroendocrinology of chronic fatigue syndrome. Endocr Rev 24: 236-252, 2003.

37. Schmidt ME, Semik J, Habermann N, Wiskemann J, Ulrich CM and Steindorf K: Cancer-related fatigue shows a stable association with diurnal cortisol dysregulation in breast cancer patients. Brain Behav Immun 52: 98-105, 2016. 